\title{
Feasibility and Outcome of Reducing Salt in Bread: A Community Trial in Southern Iran
}

\author{
Mohammad Jafari ${ }^{1}$, Mashallah Mohammadi ${ }^{2}$, Hushang Ghazizadeh ${ }^{3} \&$ Nouzar Nakhaee $^{4}$ \\ ${ }^{1}$ Health Insurance Organization, Kerman, Iran \\ ${ }^{2}$ Grain Company and Trading Services, Kerman, Iran \\ ${ }^{3}$ Kerman Province Health Center, Kerman, Iran \\ ${ }^{4}$ Neuroscience Research Center, Kerman University of Medical Sciences, Kerman, Iran \\ Correspondence: Nouzar Nakhaee, Community Medicine Neuroscience Research Center, Institute of \\ Neuropharmacology, Kerman University of Medical Sciences, Kerman, Iran. Tel: 98-34-1226-4196. Fax: \\ 98-34-1226-4198. E-mail: nakhaeen@kmu.ac.ir; nakhaeen@yahoo.com
}

$\begin{array}{lr}\text { Received: February 5, } 2016 & \text { Accepted: March 13, } 2016 \quad \text { Online Published: April 29, } 2016 \\ \text { doi:10.5539/gjhs.v8n12p163 } & \text { URL: http://dx.doi.org/10.5539/gjhs.v8n12p163 }\end{array}$

\begin{abstract}
Background: Salt is linked to hypertension, stomach cancer, kidney stone, and some other diseases. Given the harmful effect of eating too much salt, which has been reported in recent years more than ever before, culture-bound interventions are emphasized to be designed in Middle Eastern Countries in order to reduce dietary salt.
\end{abstract}

Objectives: This research was aimed at studying the feasibility of gradually reducing salt in bread and its effect on blood pressure.

Patients \& Methods: In this community trial, two cities from the southeast of Iran with similar cultural conditions were selected. In addition to the installation of educational banners and door-to-door distribution of pamphlets, salt in bread in one of the cities reduced by $40 \%$ over a 4 -week period. In the control city, only door-to-door distribution of pamphlets was carried out. Urinary sodium, systolic and diastolic blood pressure, and individuals' height and weight were measured before and 12 weeks after the intervention.

Results: The average age, weight, and height in the intervention city $(n=346)$ and control city $(n=310)$ were comparable $(\mathrm{P}>0.05)$. Perceived harm of salt was similar in both groups $(\mathrm{P}>0.05)$. ANCOVA results indicated that salt intake and post-intervention systolic blood pressure had a significantly greater reduction in the intervention group than in the control group $(\mathrm{P}<0.05)$.

Conclusion: Reduction of salt in bread by $40 \%$ was an acceptable intervention to people, which reduced urinary sodium and systolic blood pressure.

Keywords: hypertension, salt, prevention, community

\section{Introduction}

Half of the adults in the world suffer from hypertension (WHO, 2012), which is responsible for half of the deaths caused by heart attacks and strokes (Alwan, 2011). It is predicted that by 2025, the number of people suffering from this disease will increase by $60 \%$, reaching 1.6 billion (Kearney et al., 2005). In Iran, more than 68 thousand individuals aged between 25 and 64 from all over the country were included in a study. Researchers concluded that $25 \%$ and $46 \%$ of Iranians suffer from hypertension and prehypertension, respectively (Esteghamati et al., 2008).

One of the factors associated with hypertension is high salt intake (Padwl, Hackam, Khan, \& Tobe, 2016) and the health benefits of low salt diet has been well established in recent reviews (Suckling, \& Swift, 2015). In the Intersalt study, which was an international study, Yanomami as a primitive tribe was investigated. It was observed that the urinary sodium concentration of Yanomami people, who lived in Amazon, was near zero and the systolic blood pressure of males and females was 100 and 90, respectively (Rose \& Stamler, 1989). A study on chimpanzees also showed that chimpanzees' systolic blood pressure increased if salt was added to their food (He \& MacGregor, 2010). It was indicated that if salt intake in a society was reduced by 4.5 grams, systolic 
blood pressure in hypertensive and healthy individuals was reduced by 4.8 and $1.9 \mathrm{mmHg}$ respectively (Nakagawa, 2004). However, it should be noted that the level of reduction in elderly people is higher in comparison with young people (He \& MacGregor, 2010).

In Canada, researchers concluded that salt intake, which is currently $3400 \mathrm{mg}$ of sodium per day in this country, was responsible for $30 \%$ and $10 \%$ of hypertension and heart attack cases, respectively, imposing $\$ 1.4$ billion on Canadian people and government (Campbell, Willis, L'Abbe, Strang, \& Young, 2011). It was also investigated in developing countries where researchers concluded that preventive measures in the form of lifestyle modification were more effective than therapeutic measures in controlling hypertension (Ibrahim \& Damasceno, 2012). In South Africa, for example, measures such as reducing salt intake in people's diet were much more affordable and effective than performance-based measures of every single individual in preventing hypertension (Bertram, Steyn, Wentzel-Viljoen, Tollman, \& Hofman, 2012). In addition to hypertension and cardiovascular diseases, salt was also involved in the etiology of other diseases such as stomach cancer, kidney stone, osteoporosis, and indirectly obesity (Quilez \& Salas-Salvado, 2012). In a cohort study of chronic kidney disease patients in England it was shown that high salt intake could adversely affect cardiovascular disease risk (Nerbass, Pecoits-Filho, McIntyre, McIntyre, \& Taal, 2015).

Although, according to the World Health Organization (WHO), the recommended maximum salt intake is $5 \mathrm{~g}$ per day (2 $\mathrm{g}$ of sodium) (WHO, 2007), studies indicated that Iranians' salt intake was around $10 \mathrm{~g}$ per day, most of which came from bread, cheese, and fast foods (Mohammadifard et al., 2012).

In Europe, it was recently concluded that reducing the salt in people's bread was an effective lever to reduce dietary salt, and consequently hypertension (Quilez \& Salas-Salvado, 2012). Although most developed countries had their own plans for reducing salt intake (Farrand, He, \& MacGregor, 2015), as far as we know, no studies in this field have been carried out in the Middle East, and no program based on local conditions has been provided. Experts recommend that culture-bound strategies be provided to reduce salt intake in people's diet in the Middle Eastern countries as soon as possible. This study was aimed at investigating the feasibility of reducing salt in bread and its effect on people's blood pressure. The results of this study can be shared with other Middle Eastern countries.

\section{Methods}

\subsection{Setting}

This study was a community intervention trial and was conducted between March 2015 and July 2015. Unlike clinical trial, the subjects of this type of study did not suffer from a particular disease, and unlike field trial, the subjects were not included in the study individually and the study encompassed an entire city or region (Boslaugh, 2008). Two cities in Kerman province (the largest province in Iran) were selected because of the relative cultural similarity and 120 kilometers distance between them. Among the cities, one was randomly selected as the intervention group (Zarand), and the other one was chosen as the control group (Ravar). This project took 12 weeks. In this study, primary outcome and secondary outcome were individuals' blood pressure and people's sodium intake, respectively. The exclusion criteria were as follows: unwillingness to participate in the study, pregnancy, lack of informed consent, bread consumption from the places intervention was impossible. The only inclusion criterion was being over 18. Informed consent was obtained from all participants. Sample size was calculated to be 350 participants in each arm using PASS to have 80 percent power to detect a $3 \mathrm{mmHg}$ difference in blood pressure between groups at an alpha level of 0.05 . Participants were selected based on a cluster sampling scheme. Thirty households were enrolled in the study in each working day. One person over 18 years was selected from each household randomly.

\subsection{Ethics Statement}

The study protocol was approved by the ethics committee of Kerman University of Medical Sciences (KUMS). All participants provided their verbal informed consent to participate in the study. A written summary of what was to be said to the subjects was given to the trained health workers. The process of obtaining the oral informed consent was supervised by the research team. A short form which stated that the consent had been presented to the participants was signed by the subjects. According to KUMS ethics committee codes, informed consent should be in written form in clinical trials (where a drug or vaccine would be tested) or in any research in which an invasive procedure (such as blood sampling) may be performed. Since our study was not a clinical trial and no invasive procedure was made, verbal consent was accepted by EC.

\subsection{Intervention}

In the intervention city, the bakers were given a speech about the harmful effects of salt. Banners were installed 
in squares and crossroads, and posters about the harmful effects of salt were installed in all bakeries and supermarkets. The bakers' cooperation was obtained via educational workshops, and thereafter by supervision. Moreover, selecting some houses, families received a brochure about the harmful effects of salt. The main intervention in the intervention city was to gradually reduce salt intake in people's diet (bread), so that it reduced from $1.5 \%$ to $0.9 \%$ within 8 weeks. In the control city, only door-to-door distribution of educational brochures was carried out, and no other educational intervention or any intervention to reduce salt in bread was carried out. Those who were responsible for door-to-door distribution were health workers trained in a workshop.

\subsection{Measurements}

In both cities, the trained individuals went door to door in the morning. In addition to prearrangements and obtaining informed consents, an adult from each family was randomly selected. Blood pressure was measured according to standard principles (Esteghamati et al., 2008) using calibrated mercury sphygmomanometer (ALPK2, Japan). To measure salt intake (grams of sodium chloride per day), the individuals were asked to throw away the first urine after waking up and go to the nearest center for a urine sample from 8:00 to 9:00 AM (Hashimoto, Yagami, Owada, Sugawara, \& Kawamura, 2008). According to the World Health Organization guidelines (WHO, 2007) and research in various studies, this method gives acceptable results (Hashimoto, Yagami, Owada, Sugawara, \& Kawamura, 2008). Salt intake was calculated using the Kawasaki formula after laboratory measurement of sodium and urine creatinine and measurement of individuals' weight and height (Hashimoto, Yagami, Owada, Sugawara, \& Kawamura, 2008). Twelve weeks later, blood pressure and urine measurements were done again. Salt in bread was measured using the guideline of Iranian National Standards Organization before and one month after the start of the intervention (Iranian National Standards Organization, 2000).

Age, gender, educational level, history of hypertension, and history of treatment were recorded in a checklist. Using the Visual Analogue Scale (VAS), the participants were asked about perceived harm of salt regarding raising the blood pressure. The acceptability of the taste of bread with reduction in salt content was questioned from the participants in the post-intervention visit. Moreover, the bakers were asked the following open-ended question: why does their bread sometimes taste too salty?

To compare categorical variables between the two groups chi square test was used. Paired and independent $t$ tests were used to compare continuous variables within and between the two groups. The post intervention variables were compared between the two groups using ANCOVA adjusting for baseline variables. $P$ less than 0.05 was considered as significant.

\section{Results}

The participants of this study included 346 and 310 individuals in the intervention and control cities, respectively. Participation rates in the two groups were $98.8 \%$ and $88.6 \%$, respectively. Proportion of those participating in the baseline survey participated in the follow up survey were comparable in the two groups (more than 95\%). The average age, weight, and height in the intervention $(\mathrm{n}=346)$ and control groups were comparable (Table 1). Perceived harm of salt and history of hypertension were similar in both groups (Table 1). In the intervention and control groups, $13.0 \%$ and $15.2 \%$ of the participants were receiving treatment for hypertension respectively. Accordingly, all background variables, except for gender, were comparable between the two groups.

Table 1. Selected characteristics of study participants

\begin{tabular}{llll}
\hline Characteristics & $\begin{array}{l}\text { Control group } \\
(\mathrm{n}=310)\end{array}$ & $\begin{array}{l}\text { Intervention group } \\
(\mathrm{n}=346)\end{array}$ & $P$ value \\
\hline Age $($ year $)$ & $48.2 \pm 10.4$ & $49.2 \pm 12.2$ & 0.289 \\
Weight $(\mathrm{kg})$ & $68.6 \pm 13.6$ & $67.7 \pm 13.3$ & 0.361 \\
Height $(\mathrm{cm})$ & $163.2 \pm 10.4$ & $161.8 \pm 10.8$ & 0.079 \\
BMI $\left(\mathrm{kg} / \mathrm{m}^{2}\right)$ & $25.7 \pm 4.5$ & $26.0 \pm 5.3$ & 0.556 \\
Perceived harm of salt & $7.6 \pm 2.4$ & $7.9 \pm 2.0$ & 0.098 \\
Gender & & & \\
Male & $154(49.7)$ & $135(39.0)$ & 0.006 \\
Female & $156(50.3)$ & $211(61.0)$ & \\
Education & & & \\
\hline
\end{tabular}




\begin{tabular}{llll}
\hline College & $121(39.0)$ & $116(33.5)$ & 0.143 \\
Others & $189(61.0)$ & $230(66.5)$ & \\
Marital status & & & \\
Married & $259(83.5)$ & $292(84.4)$ & 0.768 \\
Others & $51(16.5)$ & $54(15.6)$ & \\
History of hypertension & & & \\
Yes & $58(18.7)$ & $66(19.1)$ & \\
No & $252(81.3)$ & $280(80.9)$ & 0.905 \\
Receiving drug therapy & & & 0.418 \\
Yes & $48(15.2)$ & $45(13.0)$ & \\
No & $262(84.8)$ & $301(87.0)$ & \\
\hline
\end{tabular}

At the beginning of the study, the mean $\pm \mathrm{SE}$ of salt intake was $8.8 \pm 0.2$ and $9.5 \pm 0.2$ in the intervention and control cities, respectively $(\mathrm{P}<0.001)$. The mean baseline systolic blood pressure was $123.2 \pm 1.0$ and $128.4 \pm 1.0$ in the control and intervention groups, respectively $(\mathrm{P}<0.001)$. The mean diastolic blood pressure in the intervention group was slightly higher than that of the control group (Table 2). After adjustment for the effect of baseline blood pressure in the two groups, ANCOVA results indicated that post-intervention systolic blood pressure had a significantly greater reduction in the intervention group than that of the control group $(\mathrm{P}=0.012)$. Such a difference was also observed in the amount of salt intake $(\mathrm{P}<0.001)$. The difference between the two groups was not significant in terms of diastolic blood pressure $(\mathrm{P}=0.50)$.

Table 2. Mean blood pressure and sodium intake measurements in control $(n=310)$ and intervention $(n=346)$ group

\begin{tabular}{llllll}
\hline \multirow{2}{*}{ Measurements } & \multirow{2}{*}{ Group } & \multicolumn{2}{l}{ Mean $\pm \mathrm{SE}$} & \multirow{2}{*}{ Adjusted mean $\pm \mathrm{SE}$} & \multirow{2}{*}{$P$ value } \\
\cline { 3 - 4 } & & Baseline & After 12-weeks & & \\
\hline \multirow{2}{*}{ Systolic blood pressure (mmHg) } & Control & $123.2 \pm 1.0$ & $122.1 \pm 1.0$ & $123.8 \pm 0.78$ & \multirow{2}{*}{0.012} \\
& Intervention & $128.4 \pm 1.0$ & $122.2 \pm 0.9$ & $121.0 \pm 0.73$ & \\
\multirow{2}{*}{ Diastolic blood pressure (mmHg) } & Control & $76.1 \pm 0.7$ & $76.1 \pm 0.6$ & $76.9 \pm 0.58$ & \multirow{2}{*}{0.500} \\
& Intervention & $79.9 \pm 0.6$ & $77.1 \pm 0.6$ & $76.3 \pm 0.55$ & \\
Salt intake (g) & Control & $9.5 \pm 0.2$ & $9.0 \pm 0.2$ & $8.80 \pm 0.18$ & \multirow{2}{*}{0.001} \\
& Intervention & $8.8 \pm 0.2$ & $7.9 \pm 0.2$ & $8.0 \pm 0.16$ & \\
\hline
\end{tabular}

\section{Discussion}

Although different studies, in recent years, indicated the effect of reducing salt intake on blood pressure, and experts emphasized the need to reduce salt intake in people's diet more than before, the effect of interventions and whether people from different cultures welcome them or not should be assessed due to cultural barriers and diversities (Newson et al., 2013).

In this study, it was shown that reducing salt in bread, as a feasible method, could lower people's blood pressure. The main strong point of the study was taking advantage of community intervention trial. The results of this type of study were more realistic due to the following reasons: 1) All participants were included into the study regardless of whether they were at high risk or not. 2) Instead of being placed in specialized environments, they were placed in real-world context of homes (Koepsell, 1998). The main limitation was that although spot urine may provide acceptable measurement for sodium intake (Tanaka et al., 2002; Hashimoto et al., 2008), the reduction in sodium intake of participants was much more valid if they had used 24-hour urinary measurements.

It appears that the per capita consumption of bread in Iran is close to that of Turkey and about twice that of European countries (about $150 \mathrm{~kg}$ per year) (Quilez \& Salas-Salvado, 2012). In Iran, the standard salt level for bread is $2 \%$ of the the flour prepared for bread making. In this study, the salt level of bread was reduced from $1.5 \%$ to $0.9 \%$. In European countries, this level is about $1.5 \%$ (Quilez \& Salas-Salvado, 2012). Studies conducted in European countries indicated that according to the people living in these countries, the optimal salt level of bread is $1.2 \%$ and a total of about $25 \%$ of salt intake in such countries was provided by bread (Quilez \& 
Salas-Salvado, 2012). It was indicated that reducing salt in bread by $25 \%$ did not have much impact on its flavor (Quilez \& Salas-Salvado, 2012). In the present study, salt in bread reduced by $40 \%$, and although, according to people, bread tasted less salty, it was acceptable. Acceptance of a marked reduction in bread salting may be due to educational campaign regarding harmful effects of salt. Since reducing salt in bread had a significant impact on its flavor, durability, and consistency, it should be considered in devising strategies for reducing salt in bread (Belz, Ryan, \& Arendt, 2012). Using salt is needed for the desirable flavor of food products and necessary for the activation of gluten, yeast control, and restriction of microbial activity (Belz, Ryan, \& Arendt, 2012). It has been proposed that the best way to decrease the salt bread was to gradually reduce it by $25 \%$ and finally $50 \%$ (Bolhuis et al., 2011). It can be, therefore, concluded that given bakers' cooperation in reducing flour salt, which was also acceptable to people, reducing salt in bread by $40 \%$ in Iran is feasible.

The bakers were asked, during an interview, about the reason for increasing salt intake. According to them, the poor quality of flour was the reason. They mentioned that they were not willing to consume excessive salt, and they had to do it in order to increase dough consistency and prevent the bread from sticking to furnaces. They also recommended that if bakers were provided with high quality flour, they would not add excessive salt.

In this study, salt reduction training along with reducing salt in bread by $40 \%$ resulted in a significant reduction in urinary sodium, which was indicative of salt intake reduction. In this community intervention, systolic blood pressure indicated a $3 \mathrm{mmHg}$ reduction (Table 1), whereas diastolic blood pressure showed no significant reduction which may be due to the narrower range of diastolic blood pressure variations. In a health promotion trial in rural areas of Ghana, salt intake reduction led to a 2.2 and $1 \mathrm{mmHg}$ reduction in the blood pressure of the intervention group, which was greater in the sixth month of follow-up than in the third month (Cappuccio, Kerry, Micah, Plange-Rhule, \& Eastwood, 2006). Observing no obvious effects on diastolic blood pressure was probably due to the short follow-up period. In Argentina, by reducing salt in bread, systolic blood pressure indicated higher reduction than diastolic blood pressure (1.66 and $0.76 \mathrm{~mm} \mathrm{Hg}$ respectively) (Ferrante, 2011).

\section{Conclusions}

Despite a $40 \%$ reduction of salt in bread, people welcomed consuming bread that tasted less salty. This study indicated that educating people along with reducing the amount of salt in bread could reduce urinary sodium and systolic blood pressure.

\section{Acknowledgements}

This study was financially supported by Iran Health Insurance Organization.

\section{Competing Interests Statement}

The authors declare that there is no conflict of interests regarding the publication of this paper.

\section{References}

Alwan, A. (2011). Global status report on noncommunicable diseases 2010. World Health Organization, Geneva.

Belz, M. C., Ryan, L. A., \& Arendt, E. K. (2012). The impact of salt reduction in bread: A review. Critical Reviews in Food Science and Nutrition, 52(6), 514-24. http://dx.doi.org/10.1080/10408398.2010.502265

Bertram, M. Y., Steyn, K., Wentzel-Viljoen, E., Tollman, S., \& Hofman, K. J. (2012). Reducing the sodium content of high-salt foods: Effect on cardiovascular disease in South Africa. Ournal South African Medical J, 102(9), 743-5. http://dx.doi.org/10.7196/samj.5832

Bolhuis, D. P., Temme, E. H., Koeman, F. T., Noort, M. W., Kremer, S., \& Janssen, A. M. (2011). A salt reduction of $50 \%$ in bread does not decrease bread consumption or increase sodium intake by the choice of sandwich fillings. Journal of Nutrition, 141(12), 2249-55. http://dx.doi.org/10.3945/jn.111.141366

Boslaugh, S. (2008). Encyclopedia of epidemiology (Vols. 1-2). Thousand Oaks, CA: SAGE Publications, Inc. http://dx.doi.org/10.4135/9781412953948

Campbell, N. R., Willis, K. J., L'Abbe, M., Strang, R., \& Young, E. (2011). Canadian initiatives to prevent hypertension by reducing dietary sodium. Nutrients, 3(8), 756-64. http://dx.doi.org/10.3390/nu3080756

Cappuccio, F. P., Kerry, S. M., Micah, F. B., Plange-Rhule, J., \& Eastwood, J. B. (2006). A community programme to reduce salt intake and blood pressure in Ghana [ISRCTN88789643]. BMC Public Health, 24(6), 13. http://dx.doi.org/10.1186/1471-2458-6-13

Esteghamati, A., Abbasi, M., Alikhani, S., Gouya, M. M., Delavari, A., Shishehbor, M. H., ... Ramezani, R. D. (2008). Prevalence, awareness, treatment, and risk factors associated with hypertension in the Iranian. American Journal of Hypertension, 21(6), 620-6. http://dx.doi.org/10.1038/ajh.2008.154 
Farrand, C., He, F. J., \& MacGregor, G. A. (2015). Reducing population salt intake in the Eastern Mediterranean Region-time for urgent action. Eastern Mediterranean Health Journal, 20(12), 761-4.

Ferrante, D., Apro, N., Ferreira, V., Virgolini, M., Aguilar, V., Sosa, M., ... Casas, J. (2011). Feasibility of salt reduction in processed foods in Argentina. Revista Panamericana de Salud Pública, 29(2), 69-75. http://dx.doi.org/10.1590/S1020-49892011000200001

Hashimoto, T., Yagami, F., Owada, M., Sugawara, T., \& Kawamura, M. (2008). Salt preference according to a questionnaire vs. dietary salt intake estimated by a spot urine method in participations at a health check-up center. Internal Medicine, 47(5), 399-403. http://dx.doi.org/10.2169/internalmedicine.47.0622

He, F. J., \& MacGregor, G. A. (2010). Reducing population salt intake worldwide: From evidence to implementation. Progress in Cardiovascular Diseases, 52, 363-382. http://dx.doi.org/10.1016/j.pcad.2009. 12.006

Ibrahim, M. M., \& Damasceno, A. (2012). Hypertension in developing countries. Lancet, 380(9841), 611-9. http://dx.doi.org/10.1016/S0140-6736(12)60861-7

Iranian National Standards Organization. (2000). Standard, (2628), 9-19.

Kearney, P. M., Whelton, M., Reynolds, K., Muntner, P., Whelton, P. K., \& He, J. (2005). Global burden of hypertension: Analysis of worldwide data. Lancet, 365(9455), 217-23. http://dx.doi.org/10.1016/S01406736(05)70151-3

Koepsell, T. D. (1998). Epidemiologic issues in the design of community intervention trials. In R. C. Brownson, \& D. B. Petitti (Eds.), Applied Epidemiology: Theory to practice (pp. 177-211). New York: Oxford University Press.

Mohammadifard, N., Fahimi, S., Khosravi, A., Pouraram, H., Sajedinejad, S., Pharoah, P., \& et al. (2012). Advocacy strategies and action plans for reducing salt intake in Iran. Archives of Iranian Medicine, 15(5), $320-4$.

Muthuri, S. K., Oti, S. O., Lilford, R. J., \& Oyebode, O. (2016). Salt Reduction Interventions in Sub-Saharan Africa. A Systematic Review, 11(3), e0149680.

Nakagawa, H. (2004). Salt reduction in a population for the prevention of hypertension. Environmental Health \& Preventive Medicine, 9(4), 123-129. http://dx.doi.org/10.1007/BF02898090

Nerbass, F. B., Pecoits-Filho, R., McIntyre, N. J., McIntyre, C. W., \& Taal, M. W. (2015). High sodium intake is associated with important risk factors in a large cohort of chronic kidney disease patients. European Journal of Clininical Nutrition, 69(7), 786-90. http://dx.doi.org/10.1038/ejcn.2014.215

Newson, R. S., Elmadfa, I., Biro, G., Cheng, Y., Prakash, V., Rust, P., ... Feunekes, G. I. J. (2013). Barriers for progress in salt reduction in the general population. An international study. Appetite, 71, 22-31. http://dx.doi.org/10.1016/j. appet.2013.07.003

Padwal, R., Hackam, D., Khan, N., \& Tobe, S. (2016). Primary prevention of CVD: Modification of diet in people with hypertension. BMJ Clininical Evidence, 5, 214.

Quilez, J., \& Salas-Salvado, J. (2012). Salt in bread in Europe: Potential benefits of reduction. Nutrition Reviews, 70(11), 666-78. http://dx.doi.org/10.1111/j.1753-4887.2012.00540.x

Rose, G., \& Stamler, J. (1989). On behalf of the INTERSALT Cooperative Research Group. The INTERSALT study: Background, methods and main results. Journal of Human Hypertension, 3, 283-288.

Suckling, R. J., \& Swift, P. A. (2015). The health impacts of dietary sodium and a low-salt diet. Clinical Medicine (London, England), 15(6), 585-8. http://dx.doi.org/10.7861/clinmedicine.15-6-585

Tanaka, T., Okamura, T., Miura, K., Kadowaki, T., Ueshima, H., Nakagawa, H., \& Hashimoto, T. (2002). A simple method to estimate populational 24-h urinary sodium and potassium excretion using a casual urine specimen. Journal of Human Hypertension, 16(2), 97-103. http://dx.doi.org/10.1038/sj.jhh.1001307

World Health Organization. (2007). Reducing Salt Intake in Populations, Report of a WHO Forum and Technical Meeting (Vol. 1, pp. 1-56). WHO: Geneva, Switzerland.

World Health Organization. (2012). World health statistics (p. 176). 


\section{Copyrights}

Copyright for this article is retained by the author(s), with first publication rights granted to the journal.

This is an open-access article distributed under the terms and conditions of the Creative Commons Attribution license (http://creativecommons.org/licenses/by/3.0/). 